\title{
CHARACTERISTICS OF THE BACKGROUND EMISSION SPECTRUM FROM A MINLATURE INDUCTIVELY-COUPLED PLASMA
}

\author{
R. N. SAVAGE and G. M. HIEFTJE \\ Indiana University, Department of Chemistry, Bloomington, IN 47405 (U.S.A.)
}

Summary. The spectral characteristics of the background radiation emitted by a miniature inductively-coupled plasma (i.c.p.) are carefully examined and methods for reducing undesirable features discussed. The complex nature of the background emission spectrum for the mini-i.c.p. indicates that careful line selection criteria and background correction procedures should be employed. Extending the torch coolant tube to the bottom of the region being observed in the plasma proved to be the most effective method for reducing undesirable spectral band features. Acute changes in background emission levels with r.f. power and nebulizer gas flow rates emphasize the need for careful control of these parameters to achieve high precision. Comparison between mini-i.c.p. and conventional i.c.p. spectra reveals the basic similarity of the two sources.

In a recent publication [1], a modified i.c.p. torch was described which was one-third smaller than a conventional unit. This new torch sustains a high-temperature plasma at low radio-frequency power levels (i.e., $<1.0 \mathrm{~kW}$ ) and consumes less than $81 \mathrm{~min}^{-1}$ of argon, resulting in significant savings for the user in both initial equipment price and operating costs. Significantly, a mini-i.c.p. operating at these reduced levels demonstrated the same high sensitivity, broad linear operating range, and simultaneous multi-element capabilities associated with conventional i.c.p. sources. However, in addition to the emission from the atoms of interest, the plasma also radiates its own background. The purpose of the present communication is to evaluate the spectral characteristics of this background emission.

Radiation emitted by the mini-plasma has been recorded using a highresolution scanning monochromator; the influence on this spectrum of several experimental parameters (i.e., coolant tube length, r.f. power and coolant and nebulizer gas flow rates) has been evaluated.

Background emission spectra from conventional i.c.p. sources have been examined by others [2-8] and their importance was recently discussed by Horlick [9]. Comparison between these conventional i.c.p. spectra and those of the mini-i.c.p. reveals the basic similarity of the two sources. 


\section{Experimental}

The experimental system and general operating procedures were the same here as previously described [1], except for the optical system which was altered slightly for spatial emission profiling studies underway in this laboratory. Typical operating conditions are outlined in Table 1.

Modified oplical desigr. Radialion emitted from the tail flame of the mini-plasma was collected by a 6 -in. $(15.2 \mathrm{~cm})$ spherically concave frontsurface mirror ( $f=40.5 \mathrm{~cm}, \mathrm{Al}-\mathrm{MgF}_{2} / 2000$ coating, Oriel Corp., Stamford, CT) held in place by a gimbal mount (Model 760, Newport Research Corp., Fountain Valley, CA). The mirror imaged the plasma at a point $34.7 \mathrm{~cm}$ from the entrance slit of a 1-m monochromator (Model HR1000, I.S.A., Inc., Nietuchen, $\mathrm{NJ}$, employing a $120 \times 140 \mathrm{~mm}$ holographic grating with 2400 grooves $\mathrm{mm}^{-1}$ and having a reciprocal linear dispersion of $0.39 \mathrm{~nm}$ $\left.\mathrm{mm}^{-1}\right)$. This image was then refocused by a plano-convex lens $(f=7.5 \mathrm{~cm}$, diameter $=2.5 \mathrm{~cm}$, fused silica, Melles Griot, Irvine, CA) onto the monochromator entrance slit, to yield a system magnification factor of 0.625 . Accordingly, slit dimensions given in Table 1 resulted in a $8-\mathbf{m m}$ high by $80-\mu \mathrm{m}$ wide observation region, centered $15 \mathrm{~mm}$ above the plasma load coil.

\section{Reagents}

Stock solutions were prepared as suggested by Dean and Rains [10], using reagent-grade salts and acids; analyte solutions were prepared by suitable dilution.

\section{Results and discussion}

Spectral features in mini-i.c.p. background emission. Figure 1 illustrates a typical mini-i.c.p. background emission spectrum, taken while a solution containing $100 \mu \mathrm{g} \mathrm{Mn} \mathrm{ml} \mathrm{m}^{-1}$ and $10 \mu \mathrm{g} \mathrm{Ca} \mathrm{ml}^{-1}$ was being nebulized. A highresolution scan such as this illustrates that the spectral background consists of three kinds of features, namely continuum, line spectra, and band spectra.

The continuum background (i.e., non-zero baseline) has been attributed mainly to bremsstrahlung radiation [5]. (Note that the spectrum in Fig. 1 has not been corrected for the wavelength-dependent response of the optical

\section{TABLE 1}

Operating conditions for background scans

R.f. power $1.0 \mathrm{~kW}$ (Forward power output from r.f. generator)

Gas flows (1 $\left.\mathrm{min}^{-1}\right)$ coolant, 7.9 ; plasma, 0.3 ; nebulizer, $0.97,1.0 \mathrm{~mm}$ (i.d.) capillary injection tube in torch (cf. Fig. 2 in [1])

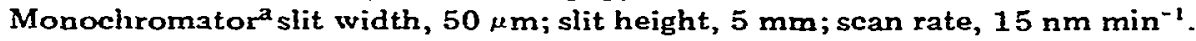

Picoammeter scale $10 \times 10^{-9} \mathrm{~A}$.

Recorder 1 in. $\min ^{-1}$.

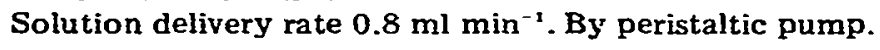

antrance slit $=$ exit slit. 
system and detector.) As detailed later, the magnitude of the continuum can change with experimental conditions, emphasizing the need for careful background-correction procedures. In general, the intensity of the continuum was low as long as the viewing zone was restricted to the plasma tail flame and did not overlap with the core [5] of the plasma.

Superimposed on the continuum were narrow spectral lincs and broadband features. The most prominent lines were those of the support gas, argon. The intensities of the 415.8-nm and 420.1-nm lines (excitation energies of approximately $14.7 \mathrm{eV}$, each) indicate the high excitation capabilities of the plasma and suggest a high density of argon metastable species [4]. Some of the more interesting lines were those of hydrogen which appear very broad. Most of the Balmer series can be seen, but by far the most intense feature in this group was the $\mathrm{H}_{\beta}$ line at $486.1 \mathrm{~nm}$. The close proximity of the $397.0-\mathrm{nm} \mathrm{H}$ line to the $396.9-\mathrm{nm}$ Ca II line could result in significant interference. The carbon line at $247.9 \mathrm{~nm}$ was assumed to arise from a still-unidentified impurity in the argon support gas.

The most troublesome spectral features in the mini-i.c.p. background were the molecular emission bands, originating from species such as NO, NH and OH. Presumably, nitrogen-containing molecules formed when air was entrained into the plasma tail flame. The $\mathrm{NO}_{\gamma}$ system present in the 200275-nm region could easily interfere with the 257.6-nm Mn II and other analyte lines (e.g., $\mathrm{Zn}, \mathrm{Cd}$ or $\mathrm{P}$ ). The $\mathrm{NH}$ band at $336.0 \mathrm{~nm}$ was also very prominent. Bands near $360 \mathrm{~nm}, 390 \mathrm{~nm}$ and $420 \mathrm{~nm}$ which degrade to the blue and the one at $610 \mathrm{~nm}$ degrading to the red were believed to arise from $\mathrm{CN}$, although those at $360 \mathrm{~nm}$ and $390 \mathrm{~nm}$ might have originated from $\mathrm{N}^{2}$ and $\mathrm{N}^{2+}$, respectively [7]. Because an aqueous solvent was used, strong $\mathrm{OH}$ bands at $306.4 \mathrm{~nm}$ and $281.1 \mathrm{~nm}$ were generated, which could interfere with elemental lines of $\mathrm{Al}, \mathrm{Be}, \mathrm{Bi}, \mathrm{Cu}, \mathrm{Mg}, \mathrm{Si}$, and $\mathrm{V}$. Background spectra similar to Fig. 1 were also obtained from a conventional i.c.p. operating in this laboratory, emphasizing the similarity of the sources.

Effect of coolant tube length on plasma background. The spectrum shown in Fig. 1 was taken from a mini-plasma sustained by a torch whose coolant tube extended $18 \mathrm{~mm}$ beyond the end of its plasma tube (cf. Fig. 2 in [1]). When the coolant tube was shortened to only $14 \mathrm{~mm}$ above the plasma tube, the background changed dramatically. In particular, the continuum level decreased to one-third that in Fig. 1 , and the $\mathrm{Ar}$ and $\mathrm{H}$ line intensities dropped. Conversely, all the band emission levels rose in intensity. The $\mathrm{NH}$, NO and $\mathrm{CN}$ bands increased sharply while $\mathrm{OH}$ rose only slightly. In addition, $\mathrm{C}_{2}$ and $\mathrm{CH}$ bands appeared. These findings agree with Truitt and Robinson [4] who reported that conventional-size torches with shorter coolant tubes sustained plasmas whose background spectra were richer in band features.

The reasons for these changes in background emission become evident when one carefully observes the size and location of the plasmas formed by the two torches. When a shorter coolant tube was employed, the optical 

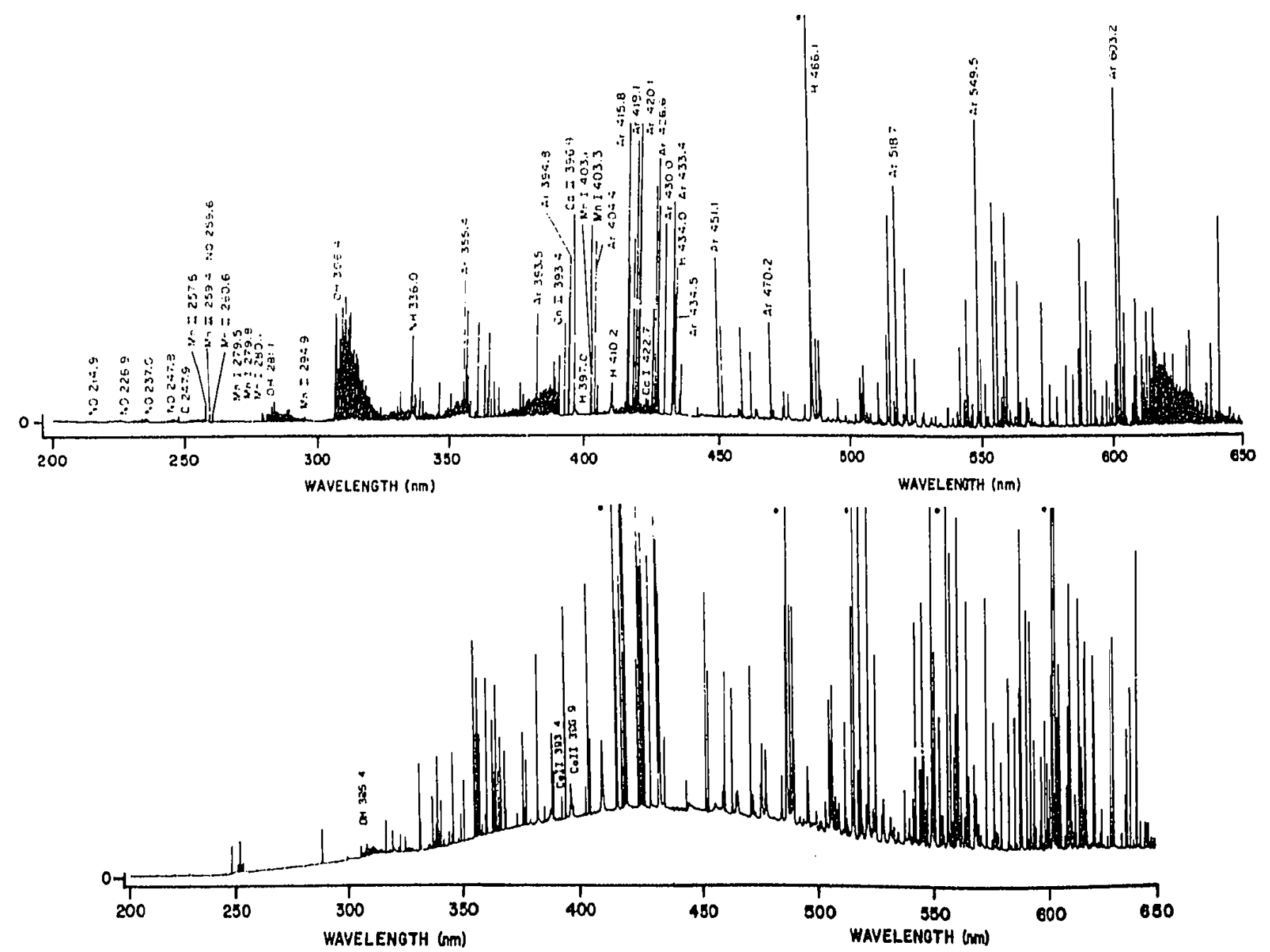

Fig. 1. Typical mini-i.c.p. background spectrum. Asterisk denotes off-scale reading; $18-\mathrm{mm}$ long coolant tube (see text for explanation).

Fig. 2. Background emission from a plasma torch having an extended coolant tube (vertical scale same as Fig. I). Asterisk denotes offscale reading; $6-\mathrm{cm}$ long coolant tube. 
system viewed a region slightly above the plasma core. In contrast, the longer torch sustained an extended plasma whose core partially fell into the monochromator viewing region. Consequently, when a longer torch was employed, a hotter region in the plasma was viewed, resulting in increased continuum and line intensities and decreased molecular formation. In addition, the longer coolant tube reduced air entrainment into the plasma, thereby lowering further the intensity of nitrogen-containing bands. Because band spectra often cause the most troublesome interferences, it seems advisable to employ torches with longer coolant tubes.

Extending this line of reasoning, it would seem appropriate to elongate the coolant tube to encompass the entire observation region. Figure 2 shows the spectrum generated by a plasma with a torch that contained a coolant tube which extended $6 \mathrm{~cm}$ above its plasma tube. Compared to Fig. 1, the spectrum in Fig. 2 (same intensity scale) has a much more intense continuum and is richer in narrow lines. However, band spectra have almost completely disappeared; only a weak $\mathrm{OH}$ band at $306.4 \mathrm{~nm}$ remains. The $\mathrm{Ca}$ and $\mathrm{Mn}$ lines appear weaker than in Fig. 1 because a solution containing only $1 \mu \mathrm{g} \mathrm{ml}^{-1}$ of each element was introduced. It is surprising that the Ca II 393.4-nm line is less intense than the Ca II 396.9-nm line; possibly a different excitation mechanism prevails in this type of plasma than in those operated conventionally.

The unusual features in the spectrum of Fig. 2 can be related to the radically different plasma sustained by the extended torch. The core of the plasma (although diluted) extends the full length of the coolant tube (i.e. $6 \mathrm{~cm}$ ) with a tail flame protruding approximately $5 \mathrm{~cm}$ beyond the end of the torch. Because the observation zone is fixed, the spectrum in Fig. 2 originates from the core of the plasma itself where higher temperatures produce more intense continuum and line spectra and enhanced molecular decomposition. In addition, atmospheric gases are completely excluded from the plasma.

An extended plasma of this nature possesses several intriguing attributes, including freedom from molecular emission and enhanced stability. Moreover, it was possible to sustain the extended mini-plasma (under the gas flow and sample introduction conditions given in Table 1) with as little as $400 \mathrm{~W}$ of r.f. power. However, even at these lower power levels, the extended quartz coolant tube fogged up quickly (i.e., within two hours of operation), causing a decrease in analyte emission intensity. Consequently, it seems prudent to extend the coolant tube only to the bottom of the observation zone but not beyond it.

Effect of r.f. power and gas flow rates on background emission. Radiofrequency power level has a profound effect on mini-i.c.p. background spectra. As r.f. power was increased, all background features intensified accordingly, demonstrating the necessity for accurate control of r.f. power during determinations. Importantly, the 396.9-nm and 393.4-nm Ca II lines each increased in intensity only $20 \%$ when the r.f. was increased from $1.0 \mathrm{~kW}$ to $1.5 \mathrm{~kW}$, while 
the background level rose over 300\%. Therefore, better signal-to-background ratios (and correspondingly, lower detection limits) can be obtained at low powers; similar conclusions were reached by Boumans and de Boer [11] for a conventional plasma.

Increasing the coolant gas flow rate from 7.9 to $9.81 \mathrm{~min}^{-1}$, caused band intensities to decrease slightly while continuum and line intensities rose. These changes result from both a reduction in air entrainment at the higher flow and also a growth in the plasma corc size. In general, these changes were small and little reduction in plasma background seemed to be gained by increasing the coolant gas flow rate.

Nebulizer gas flow also influenced the background; most features decreased in intensity when the nebulizer flow was raised from 0.97 to $1.301 \mathrm{~min}^{-1}$, presumably because of reduced temperatures in the plasma tail flame [12]. In contrast, the $\mathrm{OH}$ band was observed to intensify as aerosol flow rate was raised, probably because of the larger quantities of aerosol being delivered to the plasma. Obviously, precise control of the nebulizer flow is imperative if maximum precision is to be achieved.

This work is taken in part from the thesis of $R$. N. Savage. The work was supported in part by the National Science Foundation through grants CHE 76-10896 and 77-22152 and by the Office of Naval Research.

\section{REFERENCES}

1 R. N. Savage and G. M. Hieftje, Anal. Chem., 51 (1979) 408.

2 C. D. West and D. N. Hume, Anal. Chem., 36 (1964) 412.

3 R. H. Wendt and V. A. Fassel, Anal. Chem., 38 (1966) 337.

4 D. Truitt and J. W. Robinson, Anal. Chim. Acta, 49 (1970) 401.

5 G. F. Kirkbright and A. F. Ward, Talanta, 21 (1974) 1145.

6 J. M. Mermet, Spectrochim. Acta, Part B, 30 (1975) 383.

7 M. H. Abdallah and J. M. Mermet, J. Quant. Spectrosc. Radiat. Transfer, 19 (1978) 83.

8 V. A. Fassel, Science, 202 (1978) 183.

9 G. Horlick, Ind. Res. Dev., 20 (1978) 70.

$10 \mathrm{~J}$. A. Dean and T. C. Rains, Flame Emission and Aiomic Absorption Spectrometry, Vol. 2, M. Dekker, New York, 1971, Ch. 13.

11 P. W. J. M. Boumans and F. J. de Boer, Spectrochim. Acta, Part B, 32 (1977) 365.

12 D. J. Kalnicky, V. A. Fassel and R. N. Knisely, Appl. Spectrosc., 31 (1977) 137. 